\title{
Rate Distortion Optimized Slicing Over Bit Error Channels
}

\author{
Oztan Harmanci ${ }^{1}$ and A. Murat Tekalp ${ }^{2}$ \\ ${ }^{1},{ }^{2}$ Department of Electrical and Computer Engineering, \\ University of Rochester, Rochester, NY 14627 \\ E-mail: harmanci, tekalp@ece.rochester.edu \\ 2 also with College Engineering, \\ Koc University, Istanbul, Turkey
}

\begin{abstract}
In this paper, we study the problem of optimizing slice sizes and locations for video transmission over bit error channels. We propose a method that utilizes estimation of slice rate and distortion which is a function of the inter-macroblock dependency as exploited in the video codec. First we experimentally show that our estimation is effective. Since there are practically numerous possibilities for slice configurations and one must actually check all possibilities for a complete search, we assume segmentation of macroblocks so that a slice can only start at the segments. Although this results in a slightly suboptimal solution, it reduces the number of possibilities. However there are still practically too many configurations. Then we use the proposed RD estimation method and combine it with a dynamic programming approach to effectively determine the most optimal slice configuration. $\mathrm{RD}$ optimization is carried out in terms of minimizing the expected Lagrangian using the expected distortion of a slice. We assume that the physical layer is capable of generating an error report showing which transmission units are received in error. This allows us to use NEWPRED with macroblock level feedback and prevent wrongful decoding of erroneous macroblocks. We compare the proposed system with the two common approaches namely, fixed number of MBs per slice and fixed number of bits per slice and see that the proposed method performs better than both.
\end{abstract}

\section{INTRODUCTION}

Next generation wireless systems are commonly aiming for a TD-CDMA type of channel. In such a channel an application layer packet is transmitted as small units referred through time slots in each physical frame. In ${ }^{1}$ these are called radio link control protocol data units(PDU). Due to CDMA and channel interference these PDUs are received over a noisy channel. Unlike IP networks there is no issue of not receiving a packet. Turbo coding is used as FEC and error correction is performed. If error is not recoverable PDU retransmission is attempted for a fixed number of times. If PDU is still not succesfully transmitted the whole packet is dropped. From application layer's point of view this is a packet loss similar to IP layer loss however it is in fact not due to congestion but due to bit errors in the channel. As a result the application layer does not receive a packet with any kind of bit errors in it.

In mainstream applications and research, one slice is put in one packet. To prevent IP layer fragmentation it is considered the best approach to not to exceed the maximum transmission unit(MTU) of the physical layer since it violates Application Layer Framing(ALF), therefore slices do not exceed the MTU of the underlying network. This packet is sent through the IP network and acquires IP, UDP and RTP headers. Since header overhead is not desired packets should be as large as possible and so are the slices. It is obvious that in a packet loss network there is no point of having multiple slices in one packet in terms of error resiliency.

In this paper we assume that channel does not drop packets with bit errors in it and the application layer may receive packets with bit errors in them together with a loss report that specifies which PDUs have been received with error. Loss report is not actually needed since decoder can decode the stream until an invalid bit stream is detected. However there are significant issues in such an approach. First of all, in practical systems that is not the case. Especially $3 \mathrm{G}$ and $4 \mathrm{G}$ networks run under IP layer and in such networks it is not realistic for application layer to observe bit errors. Another issue is labeling the succesfully decoded macroblocks as "received error-free". Such a case occurs because of the high compression rates of the video codecs. For example a bit flip can occur in the macroblock $M_{i}$ but it may be detected at macroblock $M_{j}, j>i$ as a result $M_{k}, k \in\{i, \ldots, j\}$ 
will be labeled as succesfully received when in fact they are in error. This causes further error introduction to the decoder. Therefore we request error reports from network channel. Under such a scenario optimization of slice size becomes a problem. Too small slices will reduce the coding efficiency and too large slices will have low error resilience.

Over bit error channels one can put multiple slices in a packet and avoid the communiation stack overhead. To the best of our knowledge, the most relevant rate distortion optimized slicing was done in ${ }^{2}$ in which the slicing decision was done at the macroblock coding level. A slice marker is inserted if the expected Lagrangian is minimized at the current block. Although the method is shown to perform better than other methods, it does not consider the loss of encoding efficiency due to the fact the macroblocks following the current slice under test will not benefit the prediction for inter slice dependency nor they are considered during the slice insertion decision. Other optimization researches are mostly experimental such as in ${ }^{3}$ and. ${ }^{4}$

In this paper we propose a solution to slice size optimization under error reporting communication channels. We would like to analyze the expected distortion of the frame under every possible slice configurations. Since there are too many possibilities, we reduce the problem to a level where we can apply dynamic programming. We assume that the slices are allowed to be multiples of a "segment". For example in our analysis we used a segment size equal to a row of MBs. Even under such a scenario one would require $2^{H_{M B}-1}$ tests where $H_{M B}$ is the number of rows. For a CIF video one would need $2^{17}$ tests which has a high complexity. Instead we propose a method to estimate the performance of a slice and further propose a dynamic programming solution that solves the problem with extremely reduced complexity. Although we assume error reporting is available, same approach can also be used for channels with no error reports.

The rest of the paper is organized as follows. In the next section we discuss the proposed solution, in particular the dynamic programming approach that we used to solve the slicing problem. In section 3 we present the results that compare the proposed solution and the two common approaches of fixed MB and fixed bits per slice. In section 4 we discuss the conclusions.

\section{PROPOSED SYSTEM}

\subsection{Error Reports}

In a TD-CDMA system the physical frames and slots per frame can be as small as 80 bytes. On top of this, turbo coding is used even further reducing the load per PDU. We propose that the physical layer appends an error report about which PDUs are received in error. In a two wireless hop scenario this is not practical since we would require both hops' error reports but in cases such as mobile device streaming from a live content from a server on IP network the wireless hop is the last hop and the physical layer on the mobile device can generate the error report. The report is actually very small since it is $1 \mathrm{bit} / \mathrm{PDU}$.

\subsection{Partially Decoded Slices}

In a straightforward implementation one realizes that using this system we can not only get rid of the RTP/UDP/IP headers for slicing but also we are able to decode the macroblocks that are received between the resynchronization marker and before the error. We do not focus on partially decoded slices since it is not the main subject of this paper.

\subsection{Estimating the Slice Rate and Distortion}

Slice sizes and insertion of resynchronization markers pose a tradeoff between video coding efficiency and error resilience. On one hand resynchronization markers prevent further spread of error and each slice is only interdependent. On the other hand each resynchronization marker is followed by a short slice header. Although short, at low bit rates these headers can consume a significant amount of the bandwidth. Furthermore, starting a new slice disables prediction from previous macroblocks thus causing reduced encoding efficiency.

With the error reporting tool we realize that it becomes a practical problem to put multiple slices per packet where it is possible to discard the significant overhead of RTP/UDP/IP headers. Although the error resiliency property of slices is well known, there is little research on the RD optimization of slicing. The mainstream research uses experimental selection of slice size. In these works either the slice size is fixed to a number of macroblocks 
or it is fixed to a number of bits. Only RD optimized approach is ${ }^{2}$ in which a "begin a slice" decision is made at the macroblock coding level. However, this prevents the slicing to miss the global structure of the slices and as a result the loss in encoding efficiency of the macroblocks that follow the current $\mathrm{MB}$ at hand is not considered in the optimization.

Our RD optimization method that uses dynamic programming to solve the slice optimization problem in a global structure point of view. A complete slice optimization method is undoubtedly the exhaustive search method which should consider all possible combinations of slicing and then perform a rate distortion analysis based on the channel model and the decoder concealment technique. In a QCIF sized video there are 99 macroblocks. The exhaustive search method would need $2^{99-1}$ number of encodings to cover all of the cases. Certainly a more practical formulation is needed to solve the problem. Our method tries to cover the problem from both error resiliency, loss in coding efficiency and a rate distortion point of view. It is based on two assumptions which we will discuss each one separately.

First we assume that slice lengths and locations are limited so that slice length should be a multiple of a segment. In the most flexible case segment size can be $1 \mathrm{MB}$ but based on experimental observations one can set a limit to the minimum slice length and use that as a segment size. The core of algorithm does not depend on the segment size. In the following dicsussions we use one row of MBs as our segment.

In the case where a segment is one MB rows long, there are $2^{H_{m b}-1}\left(H_{m b}\right.$ is the number of rows of macroblocks in the image) possible slice configurations and the exhaustive approach would require that many full frame encodings. This will require $2^{17}$ and $2^{8}$ tests for CIF and QCIF videos respectively, which is still too much to run in practical time since all combinations of all possible slicing strategies should be considered and the frame should be encoded with each slicing configuration and decoded with the channel loss model to determine the expected distortion at the decoder side.

Let $\mathbf{C}_{i}$ denote the coding decisions for macroblock $M B_{i}, i \in\left\{1, \ldots, N_{M B}\right\} . \mathbf{C}_{i}$ includes the motion vectors, reference frame, quantization levels, coding type, resudial coefficients, etc. $\mathbf{C}_{i}$ together with reference frames buffer determines the distortion $D_{i}$ of $M B_{i}$. However $\mathbf{C}_{i}$ does not determine the rate $R_{i}$ of $M B_{i}$ since inter slice prediction is used during coding. Therefore, $R_{i}$ is a function of $\mathbf{C}_{i}$ and $\mathbf{C}_{j}$ such that $j \in\left\{i-1, i-W_{M B}, i-\right.$ $W_{M B}+1, i-W_{M B}-1 \mid \mathrm{j}$ and $\mathrm{i}$ are spatial neighbors and are in the same slice $\}$. Furthermore, the Lagrangian optimization used in selecting coding modes, motion vectors and reference frames make $\mathbf{C}_{i}$ dependent on $\mathbf{C}_{j}, j$ being same as the mentioned set. Considering the fact that this dependency holds for all macroblocks iteratively, there are two noteworthy facts i) $\mathbf{C}_{i}$ does not depend on any other macroblocks if and only if $M B_{i}$ is located at the beginning of a slice. ii) $\mathbf{C}_{i}$ depends on $\mathbf{C}_{j}, j \in\{k, \ldots, i-1\}$, where $k$ is the index of the first macroblock in the same slice as $M B_{i}$.

The iterative dependency makes it hard to determine the optimal slicing at the macroblock decision making time therefore the slicing decision must be made before the $\mathbf{C}_{i}$ decisions. However, the computational complexity of performing a full search is too high since each slice possibility must be coded and tested. Our second assumption aims to get rid of the full coding of each slice configuration and instead use a reasonable estimate. Consider slice $S_{m, n}$, where $m$ denotes the location of the slice in units of rows and $n$ denotes the length of the slice in rows. Let $R M_{k}$ denote the MB index that the $k$ th slice starts at, for $S_{m, n}$ we get $R M_{k}=m \times W_{M B}$. Let $D\left(S_{m, n}\right)$ denote the distortion of this slice: $D\left(S_{m, n}\right)=\sum_{j=m \times W_{M B}+1}^{(m+n) \times W_{M B}} D_{j}$ and $R\left(S_{m, n}\right)$ denote the rate of this slice: $R\left(S_{m, n}\right)=\sum_{j=m \times W_{M B}+1}^{(m+n) \times W_{M B}} R_{j}$. Let $F_{l}$ denote the $l$ th frame. Now lets assume we have two encodings of $F_{l}$ such that $F_{l}^{0}$ is coded as a single slice: $S_{0, H_{M B}}$, resulting in $M B_{i}^{0}, i \in\left\{1, \ldots, N_{M B}\right\} . F_{l}^{1}$ is coded as a slice at each row: $\sum_{m=0}^{H_{M B}} S_{m, 1}$, resulting in $M B_{i}^{1}$. Before we start the optimization for a given frame, we start by computing these two pictures. We assume $\mathbf{C}_{i}$ 's are independent, and end up with an estimate:

$$
\tilde{D}\left(S_{m, n}\right)=\sum_{i=m W_{M B}+1}^{(m+1) W_{M B}} D_{M B_{i}^{1}}+\sum_{j=m+1}^{m+n}\left(\sum_{i=1}^{W_{M B}} D_{M B_{i+j W_{M B}}^{0}}\right)
$$

which simply is using the first row from $F_{l}^{1}$ and the rest from $F_{l}^{0}$ as an estimate for the slice $S_{m, n}$. We can also replace $\tilde{D}$ with $\tilde{R}$. 

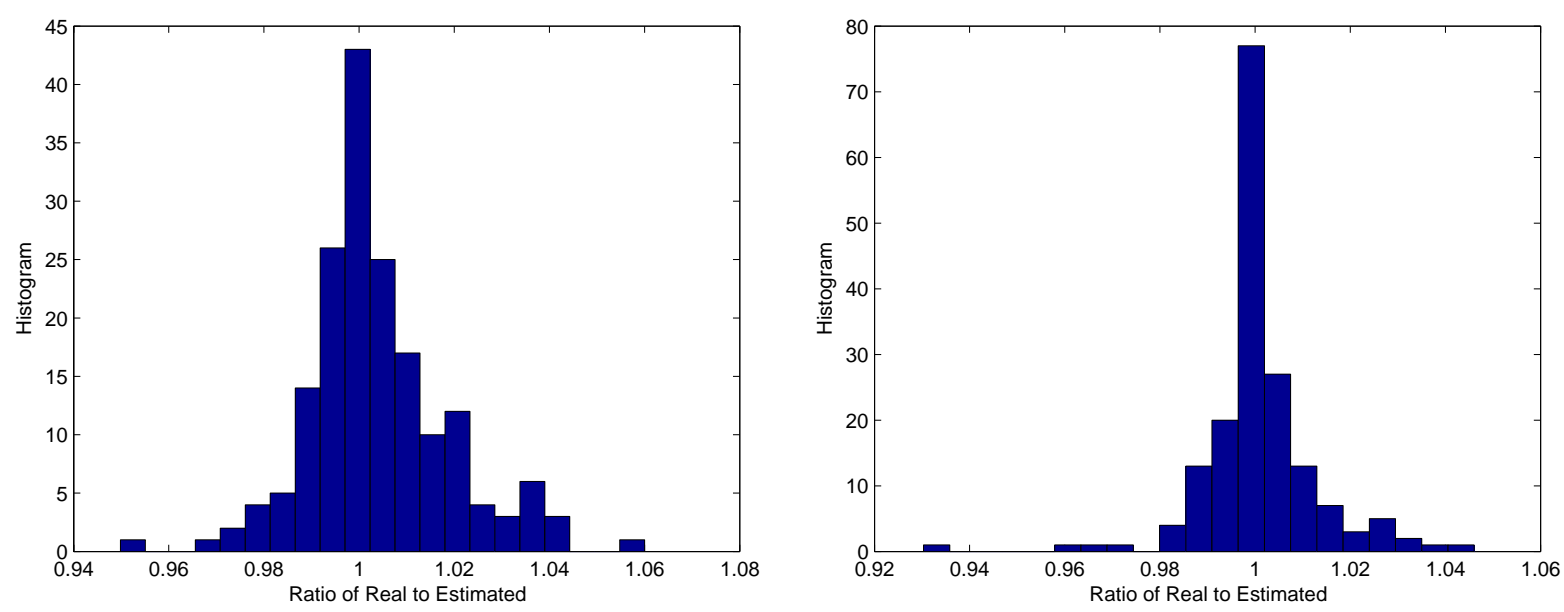

Figure 1. Testing the independence assumption: Letf pane: Histogram of ratio of estimated to actual bitrate of randomly selected slices fo random lengths at random locations. Right pane: Same as left pane except for SSD

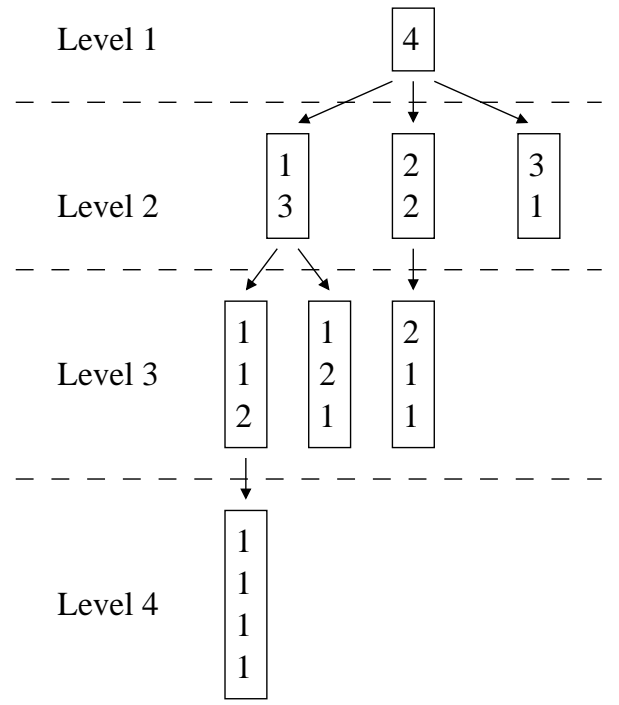

Figure 2. Slice configuration tree with 4 levels that covers all possible slices. The performance of the nodes $\{1,1,2\}$ and $\{2,2\}$ are compared and the one with the worse performance is pruned therefore saving one node in this example. The savings grow larger according to the formulae $2^{N-1}$ vs. $1+N(N-1) / 2$ where $N$ is the maximum level of the tree.

We stated earlier that we incorporate the dependence of slices but in equation 1 it may look like we are contradicting ourselves. However, note that we are in fact considering the dependence during the calculation of $F_{l}^{0}$.

We have tested the accuracy of the proposed assumption by selecting $180 S_{m, n}$ slices of random length and random locations. Figure 1 shows the resulting comparison of $\tilde{D}\left(S_{m, n}\right)$ vs. $D\left(S_{m, n}\right)$ and $\tilde{R}\left(S_{m, n}\right)$ vs. $R\left(S_{m, n}\right)$ in terms of error percentage. Both variables are predicted effectively.

\subsection{Rate Distortion Optimized Slicing}

Considering the fact that there are many valid combinations of slices, the determination of the optimal slice configuration becomes too complex depending on the segment size and the complexity of concealment method used. Note that a valid configuration is any confguration that satisfies $\sum n=H_{M B} \mid S_{m, n} \in F_{l}$ which means there are $2^{H_{M B}-1}$ possible configurations. To quickly eliminate the combinations we use a tree structured dynamic 
programming method. Let $\mathbf{S} \in \mathbb{Z}^{+H_{M B}}$ denote a possible slicing configuration. $i$ th element of $\mathbf{S}$ is denoted with $\mathbf{S}(i)$ and it represents the length of $i$ th slice. Tree's top node starts with $\mathbf{S}=H_{M B}$ and it is the 1st level meaning that it is composed of only 1 slice. The second level is composed of 2 slices, 3rd level 3 and so forth. $i$ th level nodes are created from $i-1$ st level nodes. A node with configuration $\mathbf{S}$ at $i$ th level generates $S(i)-1$ new nodes by the following rule: $\forall k \in\{1, \ldots, S(i)-1\}, \mathbf{S}_{k}=\{S(1), \ldots, S(i)-1, k, S(k)-1\}$ and add $\mathbf{S}_{k}$ as the child node of $\mathbf{S}$. Recursively do this and build the tree until $H_{M B}$ th level is achieved which has only one node. Also note that not all nodes grow until $H_{M B}$ th level. Figure 2 presents such a full grwon tree for a 4-level scenario.

This tree is basically useless on its own since it includes all of the possibilities. To perform quick purging of the tree nodes, we calculate the estimated coding and store the rate and expected distortion of the slices at the nodes at the build time. We perform the purging as follows: before a node with configuration $\mathbf{S}_{a}$ at level $l$ is used to spawn new nodes, it's performance is compared to other already calculated nodes if the configurations match: A node $\mathbf{S}_{b}$ at level $k$, is compared with $\mathbf{S}_{a}$, if $\sum_{i=1}^{k-1} \mathbf{S}_{b}(i)=\sum_{i=1}^{l-1} \mathbf{S}_{a}(i)$. The node with worse performance is not further used to spawn new nodes. For example in figure 2 , nodes $\{1,1,2\}$ and $\{2,2\}$ are such comparable nodes. The one with worse performance should not be further considered including its children nodes.

The comparison metric is the minimization of the Lagrangian $L=E[D]+\lambda_{M B} R$. This is possible since the slices are independently decodable. As a result we end up with $1+\frac{H_{M B} \times\left(H_{M B}-1\right)}{2}$ semi-complete loss simulations instead of $2^{H_{M B}-1}$ full simulations. $\lambda_{M B}$ is same as the one used in selecting the optimal MB mode as described in. ${ }^{5}$

To perform RD optimization in a lossy environment, we use $E[D]$ instead of $D$ to determine the slice distortion. We assume that the decoder concealment strategy is known at the encoder as well as the channel loss model. As a result we can predict the expected distortion of a given slice by simply estimating the length of the bits using the formula 1 for $R(\cdot)$. For $M B_{i}$ that is located in the slice beginning at $R M_{k}$, the number of bits since the slice header is $R_{\text {cum }_{i}}=\sum_{i=R M_{k}}^{i} R_{i}$. Number of PDUs that are used to transmit until $M B_{i}$ is $N_{P D U_{i}}=R_{c u m_{i}} / L_{P D U}$, therefore loss probability of $M B_{i}$ is $p_{i}=1-\left(1-p_{p d u}\right)^{N_{P D U_{i}}}$ where $p_{p d u}$ is the loss probability of a PDU. If $\tilde{D}_{i}$ denotes the concealment distortion, then the expected distortion of the $M B_{i}$ is $E\left[D_{i}\right]=p_{i} \tilde{D}_{i}+\left(1-p_{i}\right) D_{i}$. E[D] of slice $S_{m, n}$ is equal to $\sum_{i}\left(E\left[D_{i}\right] \mid i \in S_{m, n}\right)$

\subsection{Using Smaller Segments for Better Accuracy}

In the previous discussions we used segments as the size of a row of macroblocks. The proposed algorithm can be extended to smaller segments as follows. For example for $W_{M B} / 2$ sized segments, we generate another frame: $F_{l}^{2}$ similar to $F_{l}^{0}$ and $F_{l}^{1}$, but the slices start at middle of the rows instead of at the beginning of the rows. The generated slices are still $W_{M B}$ long. However we realize that one does not have to completely encode $W_{M B}$ slices since the second half of MBs of slices in $F_{l}^{2}$ are already encoded in first half of MBs of slices in $F_{l}^{1}$. This is because of the fact that the second half of a slice in $F_{l}^{2}$ has exactly the same predictive coding neighborhood as the MBs in first half of slices in $F_{l}^{2}$. As a result one needs to only encode one half of a frame in $F_{l}^{2}$.

This will allow for estimating the distortion and bitrate for slices that are more free in the size and location. Note that using smaller segments does not only mean testing smaller slices but also testing various slice configurations that can begin and end at the middle of the rows for example.

The tree structure and pruning would stay the same and the formulae proposed in the previous sections can be applied with a similar approach. For $W_{M B} / 2$ sized segments, the exhaustive approach would require $2^{35}$ tests while the proposed tree method requires $1+36 \times 35 / 2$ for CIF resolution. In our experiments we used QCIF sized videos therefore slices smaller than a row of macroblocks would be unnecesary. However this is just an assumption based on experimentation and needs to be justified.

\section{RESULTS}

The experimental setup is the following: 40Byte PDUs are used over a uniform PDU loss channel at two different uniform loss rates: 0.005 and 0.01 . We used low delay rate control with ACK mode NEWPRED using per MB feedback over a $96 \mathrm{kbps}$ channel with $10 \mathrm{~Hz}$ QCIF video. We performed 50 experiments at each point and averaged the frame pSNR's of the Y channel. We used H264 in high complexity mode as described in. ${ }^{6}$ Previous frame copy is used for concealment for its simplicity. 

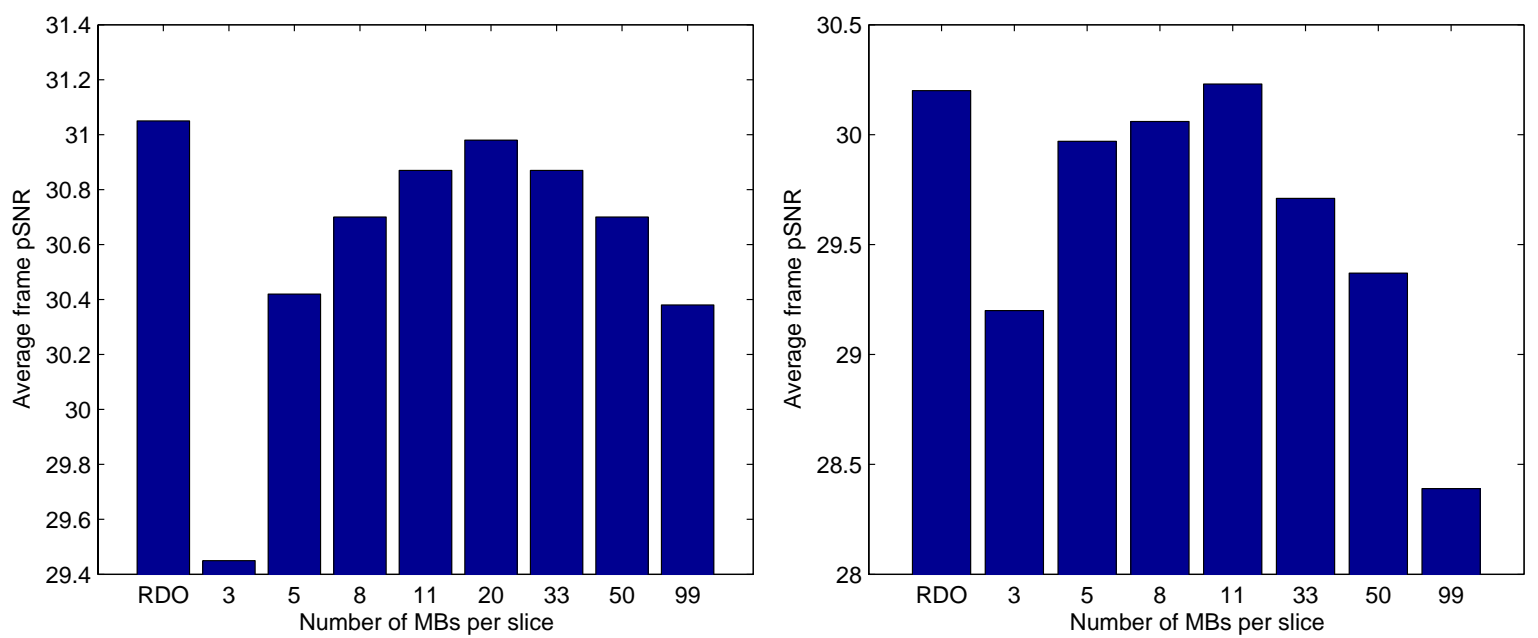

Figure 3. Left pane: Comparison of average frame pSNR of proposed system with fixed MB per slice at 0.005 PDU error rate for the sequence Foreman. Right Pane: at 0.01 PDU error rate
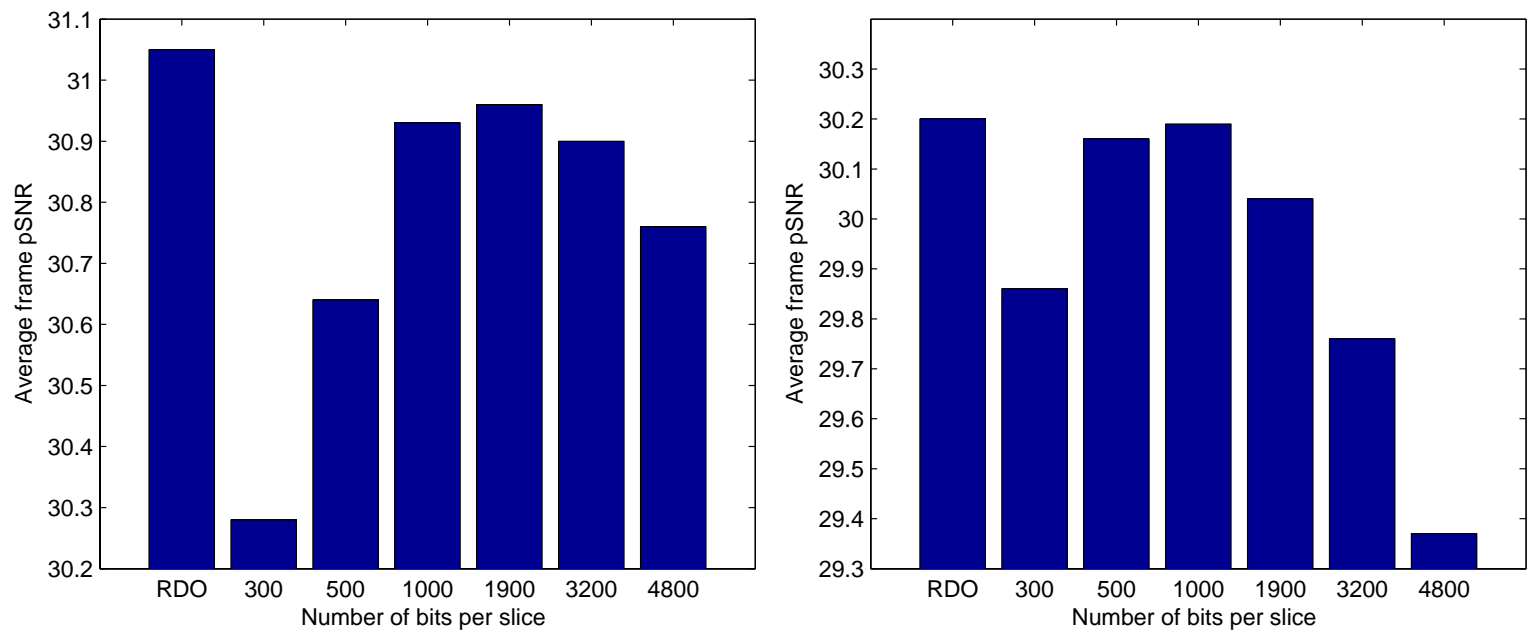

Figure 4. Left pane: Comparison of average frame pSNR of proposed system with fixed bits per slice at 0.005 PDU error rate for the sequence Foreman. Right Pane: at 0.01 PDU error rate

$\mathrm{In}^{2}$ the authors choose to compare their system with common systems such as random intra update and fixing the slices to the beginning of group of blocks(GOB). Both these approaches are non-adaptive and can be extremely sub optimal considering different channel conditions and source properties. Also random intra updates can be combined with adaptive slicing to make for an even better scheme therefore it does not constitute a fair comparison. Therefore we choose to compare the proposed slicing scheme with the optimal of two schemes: fixed MBs per slice and fixed bits per slice. Furthermore we do not depend on an optimization method to determine the optimal operation paramters of the two schemes but we experimentally perform exhaustive searches.

First we compared the proposed system with fixed number of MBs per slice. Figures 3 and 5 show the performance of proposed system (marked with $R D O$ ) and various fixed MB approaches for Foreman and Carphone sequences respectively. We see that the proposed system performs slightly better than the experimentally found optimal point of fixed MB approach.

Putting fixed bits per slice is another common suggestion with which we compared our approach. Figures 4 and 6 show the results. The proposed approach operates either at the experimentally found optimal points or better. We believe that if we had uses smaller segments than the row of MBs as in our experiments, we would 

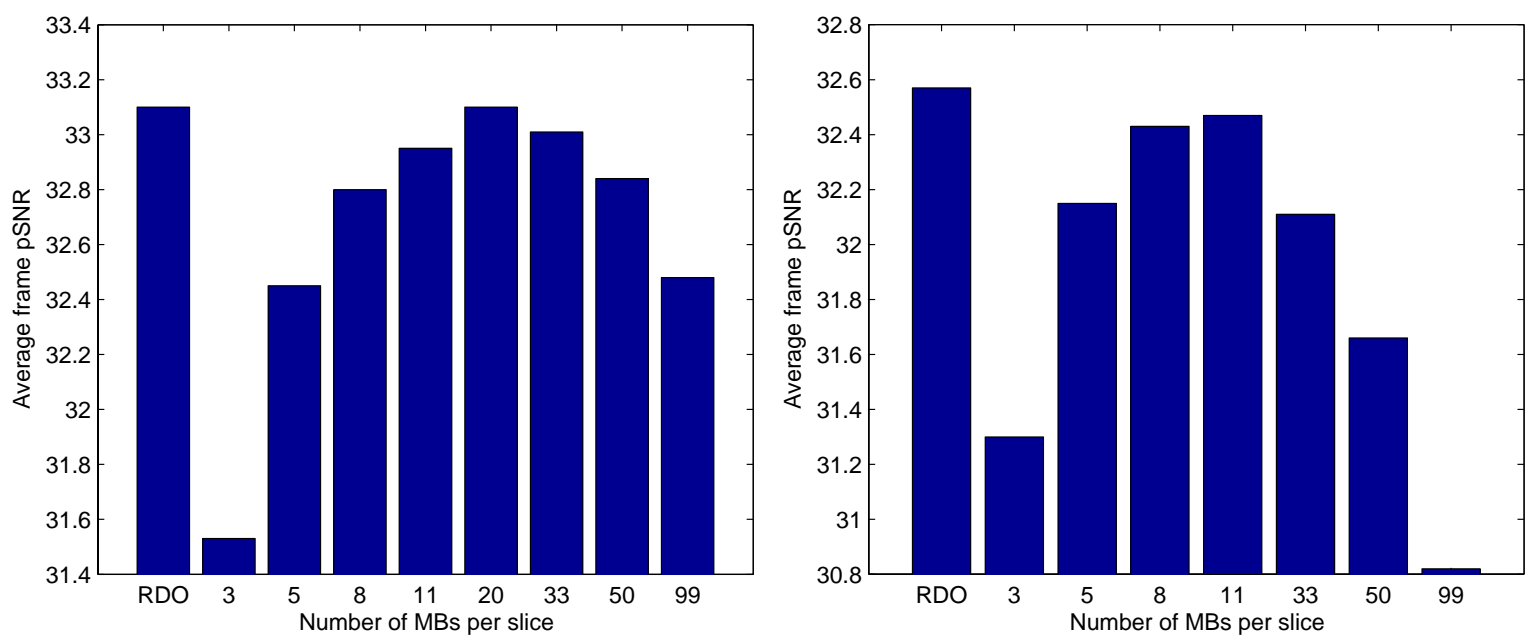

Figure 5. Left pane: Comparison of average frame pSNR of proposed system with fixed MB per slice at 0.005 PDU error rate for the sequence Carphone. Right Pane: at 0.01 PDU error rate
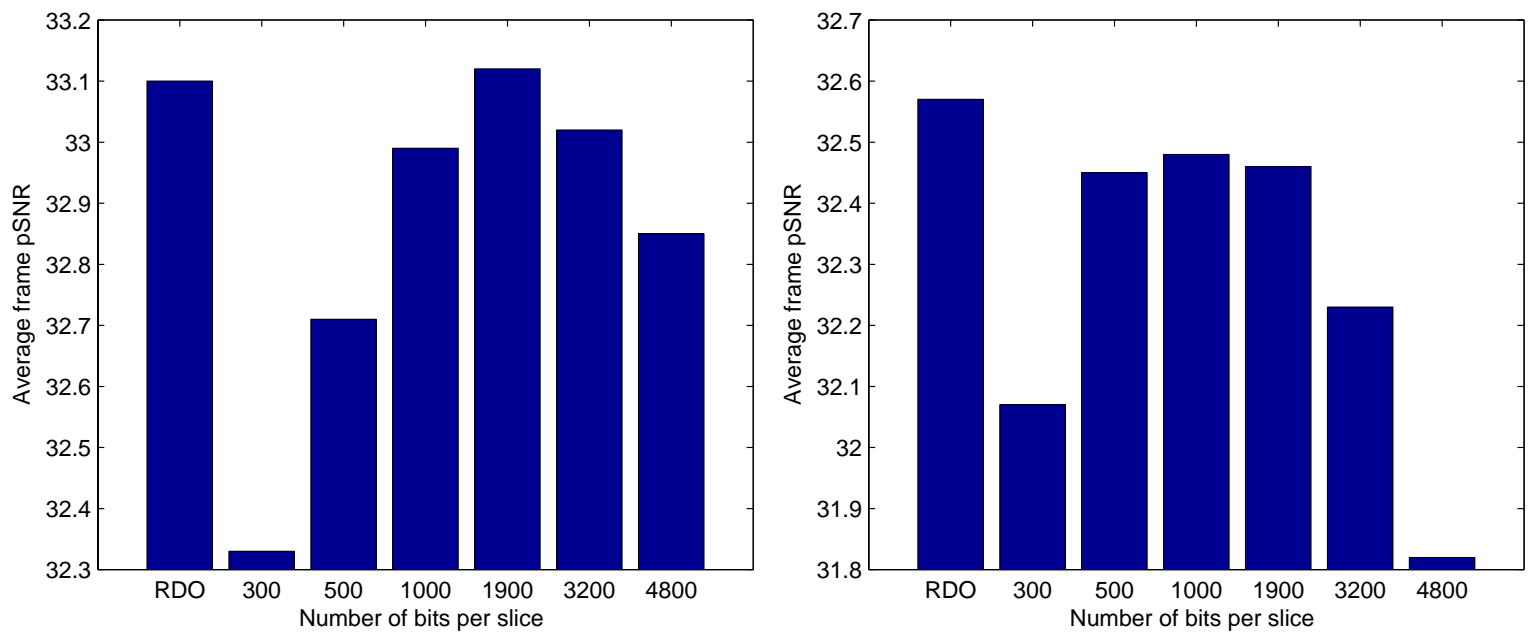

Figure 6. Left pane: Comparison of average frame pSNR of proposed system with fixed bits per slice at 0.005 PDU error rate for the sequence Carphone. Right Pane: at 0.01 PDU error rate

have ended up with superior performance.

\section{CONCLUSIONS}

In this paper we proposed a solution for rate distortion optimized determination of slice sizes and locations for error resilient video communications over bit error networks. We study the system on next generation networks and to avoid dealing with bit errors at the application layer we propose that the physical layer generate an error report that we can use at the application layer. Although this report is not a part of the RD optimization process, it allows for robust MB decoding and MB level NEWPRED feedback. The proposed method determines optimal slicing at segment resolution.

Since there a renumerous possibilities to search over to avoid encoding all such possibilities we propose a method to estimate the dependency that is used in encoding a MB. We experimentally see that our estimation is accurate enough. Then we propose our dynamic programming solution that allows us to significantly reduce the search space that finds the optimal configuration. We perform experiments on two different videos at two 
different channel conditions. Our experiments show that our method consistently performs better than the two common approaches, namely fixed bits per slice and fixed MBs per slice.

\section{REFERENCES}

1. T. Stockhammer, M. M. Hannuksela, and T. Wiegand, "H.264/avc in wireless environments," IEEE CSVT , July 2003.

2. G. Cote, S. Shirani, and F. Kossentini, "Optimal mode selection and synchronization for robust video communications over error-prone networks," IEEE JSAC, June 2000.

3. I. E. G. Richardson and M. J. Riley, "Varying slice size to improve error tolerance of mpeg video," Proceedings of SPIE, Vol. 2668, p. 365-371, 1996.

4. R. Talluri, "Error resilient video coding in the mpeg-4 standard," IEEE Communications Magazine, June 1998.

5. T. Wiegand, H. Schwarz, A. Joch, F. Kossentini, and G. J. Sullivan, "Rate-constrained coder control and comparison of video coding standards," IEEE CSVT, July 2003.

6. I. J. JVT-I049, "Joint model reference encoding methods and decoding concealment methods." 